\title{
Nomogram for Predicting Anastomotic Leakage after Rectal Cancer Surgery in Elderly Patients with Dysfunctional Stomata
}

This article was published in the following Dove Press journal: Cancer Management and Research

\author{
Chuangkun $\mathrm{Li}^{1,2, *}$ \\ Weiwen Liang ${ }^{1,2, *}$ \\ Lili Chu ${ }^{1, *}$ \\ Yingqi Wei ${ }^{1,2}$ \\ Xiusen Qin ${ }^{1,2}$ \\ Zifeng Yang ${ }^{1,2}$ \\ Wentai Guo ${ }^{1,2}$ \\ Hui Wang ${ }^{1,2}$ \\ Huaiming Wang ${ }^{1,2}$ \\ Rongkang Huang ${ }^{1,2}$ \\ 'Department of Colorectal Surgery, Sixth \\ Affiliated Hospital, Sun Yat-Sen \\ University, Guangzhou, Guangdong, \\ 510655, People's Republic of China; \\ ${ }^{2}$ Guangdong Provincial Key Laboratory of \\ Colorectal and Pelvic Floor Diseases, \\ Sixth Affiliated Hospital, Sun Yat-Sen \\ University, Guangzhou, Guangdong, \\ 510655, People's Republic of China \\ *These authors contributed equally to \\ this work
}

Purpose: Anastomotic leakage after rectal cancer surgery in elderly patients is a critical challenge. Many risk factors have been found and many interventions tried, but anastomotic leakage in elderly patients remains difficult to deal with. This study aimed to create a nomogram for predicting anastomotic leakage after rectal surgery in elderly rectal cancer patients with dysfunctional stomata.

Methods: We collected data from 326 consecutive elderly patients with dysfunctional stomata after rectal cancer surgery at the Sixth Affiliated Hospital, Sun Yat-Sen University from January 2014 to December 2019. Risk factors of anastomotic leakage were identified with multivariate logistic regression and used to create a nomogram. Predictive performance was evaluated by the area under the receiver-operating characteristic (ROC) curve.

Results: American Society of Anesthesiologists score $\geq 3$, male sex, and neoadjuvant radiotherapy were identified as significantly associated factors that could be combined for accurate prediction of anastomotic leakage on multivariate logistic regression and development of a nomogram. The area under the ROC curve for this model was 0.645 . The $C$-index value for this model was 0.645 , indicating moderate predictive ability of the risk of anastomotic leakage.

Conclusion: The nomogram showed good ability to predict anastomotic leakage in elderly patients with rectal cancer after surgery, and might be helpful in providing a reference point for selection of surgical procedures and perioperative treatment.

Keywords: nomogram, rectal cancer, anastomotic leakage, elderly patients

\section{Introduction}

Colorectal cancer (CRC) is one of the most common cancers. ${ }^{1}$ Many treatments have been reported for $\mathrm{CRC}$, but surgery is the principal means of achieving a radical effect. ${ }^{2}$ However, there are many surgical complications, especially for elderly patients. Anastomotic leakage (AL) is regarded as the leading cause of morbidity and mortality in colorectal cancer surgery, with a rate of $3 \%-21 \%{ }^{3}$

AL not only causes serious perioperative consequences, such as pelvic abscesses, peritonitis, septic shock, and even death, but is also the main risk factor of long-term complications, such as postoperative anastomotic stenosis and bowel dysfunction. ${ }^{4,5}$ Although surgical techniques and technologies and perioperative care have greatly evolved over the last several decades, AL is still the main challenge, especially for elderly patients. Compared with open surgery, laparoscopic surgery can significantly reduce the incidence of AL; however, there is no
Rongkang Huang

Department of Colorectal Surgery, The

Sixth Affiliated Hospital, Sun Yat-Sen

University, Guangzhou, Guangdong,

People's Republic of China

Tel +86-I34-802I-8647

Email huangrk3@mail.sysu.edu.cn 
difference in the AL incidence between the techniques. ${ }^{6}$ Factors that may impact the risk of AL after surgery are the experience of the surgeon performing the procedure and hospital volume, not the nuances of rectal cancer surgery. ${ }^{7}$ It has been reported that the $\mathrm{AL}$ death rate is $0.8 \%-27 \%$ after CRC surgery. ${ }^{8}$ There is a high frequency of selective stoma to prevent potential severe consequences of AL after CRC surgery in elderly patients, reflecting poor predictive ability of surgeons to identify AL risk in elderly patients. ${ }^{9,10}$ We focused on patients aged $>70$ years. According to 2018 recommendations of experts on rectal cancer in the elderly ${ }^{11}$ and guidelines for perioperative management of elderly CRC patients in China in $2020,{ }^{12}$ the average detection age of rectal cancer has reached 70 years and $50 \%$ of newly added patients with rectal cancer are aged $>70$ years.

Therefore, the development of a prediction model for AL in elderly patients after rectal surgery and determination of the risk-reduction factors in controllable strategies are very important. Risk factors of AL have been extensively studied, and common ones mentioned are male sex, age, high American Society of Anesthesiologists (ASA) score, comorbidities, emergency operation, and preoperative radiotherapy. ${ }^{13-15}$ Many authors have tried to compose models to predict AL that made sense in terms of treatment decisions and risk assessment. ${ }^{16-18}$ However, there have been few studies on predicting the rate of $\mathrm{AL}$ in elderly patients after CRC surgery. This study thus aimed to construct a nomogram for predicting the incidence of postoperative AL after CRC surgery in elderly patients. Eventually, the nomogram could be applied by clinician to achieve the goal of individualized management and precise treatment for elderly patients with rectal cancer and improve prognosis and quality of life while improving their survival rate. We present the protocol in accordance with TREND (Transparent Reporting of Evaluations with Nonrandomized Designs) checklist.

\section{Methods}

\section{Patients}

From January 2014 to December 2019, we collected data from 326 consecutive elderly patients after rectal cancer surgery at the Sixth Affiliated Hospital, Sun Yat-Sen University. We defined elderly patients as being aged $\geq 70$ years. Inclusion criteria were age $\geq 70$ years, tumors less than $15 \mathrm{~cm}$ from the anus and pathologically confirmed as rectal adenocarcinoma, and had undergone rectal cancer surgery. Exclusion criteria were abdominal metastases and those who had undergone Hartmann's surgery or abdominoperineal resection (Miles). Finally, 326 patients were included. The patients were divided into two groups: those with AL and those without.

All surgeries were performed by a clinical colorectal surgeon. Tumor staging was determined by The American Joint Committee on Cancer staging system (eighth edition). ${ }^{19}$ All patients received a complete preoperative assessment, including physical and laboratory examinations, colonoscopy with biopsy, thoracic and abdominal computed- tomography scan, and pelvic magnetic resonance imaging. The guidelines of the National Comprehensive Cancer Network were used for perioperative management. This retrospective study was approved by the ethics committee of the Sixth Affiliated Hospital, Sun Yat-Sen University.

\section{Definition of $A L$ and Follow-Up}

In this study, AL was defined as damage to the integrity of the intestinal wall of the anastomotic site due to necrosis or abscess formation, resulting in intraperitoneal and extracellular communication, which was diagnosed according to the 2010 protocol of the International Study Group of Rectal Cancer. AL was classified into three grades: A, only radiological evidence of $\mathrm{AL}$, without any required treatment; B, AL with clinical symptoms requiring either antibiotics or drainage; and $\mathrm{C}$, symptomatic $\mathrm{AL}$ requiring a return to hospital and a second operation. AL was diagnosed by meeting the criteria for grades $\mathrm{A}, \mathrm{B}$ or $\mathrm{C}$ in the present study. ${ }^{20}$

\section{Variables}

Variables analyzed as risk factors were AL were sex, age, $\mathrm{BMI}$, comorbidity, ASA score, preoperative $\mathrm{Hb}$, preoperative albumin, neoadjuvant chemotherapy, neoadjuvant radiotherapy, surgical method, operation time, pathological $\mathrm{T}$ stage, pathological $\mathrm{N}$ stage, metastasis, distance from anal margin, and anastomosis method.

\section{Statistical Analysis}

IBM SPSS 25.0 was used for data analysis. Data are expressed as means \pm SD or medians (range). MannWhitney rank-sum tests or $t$-tests were used to compare data between groups. Logistic regressions were used to analyze univariate and multivariate factors. Variables with $P<0.1$ on univariate analysis were included in the 
Table I Patient characteristics

\begin{tabular}{|c|c|c|c|}
\hline & AL, n (\%) & No $A L, n$ (\%) & $P$ \\
\hline Patients, $\mathrm{n}$ & $63(19.33 \%)$ & 263 (80.67\%) & - \\
\hline Age (years) & & & 0.667 \\
\hline$<75$ & 39 (61.90\%) & I 55 (58.94\%) & \\
\hline$\geq 75$ & $24(38.10 \%)$ & $108(41.06 \%)$ & \\
\hline Sex & & & 0.003 \\
\hline Male & $53(84.13 \%)$ & I 77 (67.30\%) & \\
\hline Female & $10(15.87 \%)$ & $86(32.70 \%)$ & \\
\hline $\mathrm{BMI}\left(\mathrm{kg} / \mathrm{m}^{2}\right)$ & $22.40 \pm 3.11$ & $22.20 \pm 3.17$ & 0.661 \\
\hline Comorbidity & 31 (49.2I\%) & $90(34.22 \%)$ & 0.035 \\
\hline ASA score & & & 0.010 \\
\hline I or 2 & 49 (77.78\%) & $243(92.40 \%)$ & \\
\hline$\geq 3$ & $14(22.22 \%)$ & $20(7.60 \%)$ & \\
\hline Preoperative $\mathrm{Hb}(\mathrm{g} / \mathrm{dL})$ & $118.70 \pm 17.39$ & $120.93 \pm 16.84$ & 0.323 \\
\hline Preoperative albumin $(\mathrm{g} / \mathrm{L})$ & $39.73 \pm 4.26$ & $39.29 \pm 4.06$ & 0.319 \\
\hline Neoadjuvant chemotherapy & 21 (33.33\%) & $68(25.86 \%)$ & 0.258 \\
\hline Neoadjuvant radiotherapy & $10(15.87 \%)$ & $23(8.75 \%)$ & 0.154 \\
\hline Surgical approach & & & 0.198 \\
\hline Laparoscopy & 55 (87.30\%) & $245(93.16 \%)$ & \\
\hline Open & $8(12.70 \%)$ & I8 (6.84\%) & \\
\hline Surgical method & & & 0.539 \\
\hline Dixon & $40(63.49 \%)$ & 179 (68.06\%) & \\
\hline Parks and Nose & $23(36.51 \%)$ & $84(31.94)$ & \\
\hline Operation time & $291.92 \pm 90.22$ & $268.15 \pm 88.59$ & 0.083 \\
\hline Pathological T stage & & & 0.034 \\
\hline $\mathrm{TI}-\mathrm{T} 2$ & 15 (23.8I\%) & 99 (37.64\%) & \\
\hline T3-T4 & 48 (76.19\%) & $164(62.36 \%)$ & \\
\hline Pathological $\mathrm{N}$ stage & & & 0.018 \\
\hline No & 34 (53.97\%) & 190 (72.24\%) & \\
\hline $\mathrm{NI}$ & $20(31.75 \%)$ & $56(21.29 \%)$ & \\
\hline N2 & $9(14.28 \%)$ & $17(6.46 \%)$ & \\
\hline Metastasis & & & 0.147 \\
\hline Yes & 7 (II.II\%) & 13 (4.94\%) & \\
\hline No & $56(88.89 \%)$ & $250(95.06 \%)$ & \\
\hline Distance from anal verge $(\mathrm{cm})$ & & & 0.327 \\
\hline$>5$ & 32 (50.79\%) & $15 \mid$ (57.4I\%) & \\
\hline$\leq 5$ & 31 (49.2।\%) & II 2 (42.59\%) & \\
\hline Anastomosis method & & & 0.480 \\
\hline Stapled & $5 \mathrm{I}(80.95 \%)$ & $202(76.81 \%)$ & \\
\hline Hand-sewn & $12(19.05 \%)$ & 61 (23.19\%) & \\
\hline
\end{tabular}


multivariate model.Forest plots were constructed with GraphPad Prism 7.

The nomogram was validated internally through 1,000 bootstrap resampling to calculate the estimated Harrell concordance index ( $C$ index), thus indicating the performance of the model. In addition, we used a calibration curveto representthe relationship between observed frequency and predicted probability.Predictive performance was evaluated by the area under the receiver-operating characteristic (ROC) curve (AUC). Differences were considered statistically significant at $P<0.05$.

\section{Results}

\section{Patients}

Characteristics of patients are shown in Table 1. A total of 326 elderly patients who had undergone rectal cancer surgery were included. Of these, 63 (19.33\%) developed AL and 53 (84.13\%) were men, the latter being significantly higher than in those without $\mathrm{AL}(P=0.003)$.
Compared to patients without AL, those with AL had poor ASA scores ( $\geq 3)$, more at T3 and T4 stages (76.19\%), higher prevalence of N1 and N2 (46.03\%).

\section{Univariate and Multivariate Analysis}

Univariate and multivariate logistic regression analyses are presented in Table 2. On univariate analysis, sex, comorbidity, ASA score, operation time, pathological $\mathrm{T}$ stage, and pathological $\mathrm{N}$ stage were associated with AL. Multivariate analysis showed that sex (HR 0.339, 95\% CI 0.144-0.802; $P=0.014$ ), ASA score $\geq 3$ (HR 3.250, 95\% CI 1.310-8.064; $P=0.011)$, and neoadjuvant radiotherapy (HR 2.876, 95\% CI 1.030-8.032; $P=0.044$ ) were independent risk factors of AL. On the basis of these results, we developed a forest plot (Figure 1).

\section{Nomogram for $\mathrm{AL}$}

The nomogram was developed to predict the risk of AL after rectal cancer surgery based on the three independent factors on multivariate logistic regression analysis, and

Table 2 Univariate and multivariate logistic regression model for risk factors of $A L$

\begin{tabular}{|c|c|c|c|c|}
\hline & & \multirow{2}{*}{$\frac{\text { Univariate }}{P}$} & \multicolumn{2}{|l|}{ Multivariate } \\
\hline & & & HR (95\% Cl) & $\boldsymbol{P}$ \\
\hline Sex & Male vs female & 0.010 & $0.339(0.144-0.802)$ & 0.014 \\
\hline Age (years) & $\geq 75$ vs $<75$ & 0.666 & & \\
\hline BMI $\left(\mathrm{kg} / \mathrm{m}^{2}\right)$ & & 0.660 & & \\
\hline Comorbidity & Absent vs present & 0.028 & $1.806(0.886-3.670)$ & 0.102 \\
\hline ASA score & I or 2 vs $\geq 3$ & 0.001 & $3.250(1.310-8.064)$ & 0.011 \\
\hline Preoperative $\mathrm{Hb}(\mathrm{g} / \mathrm{dL})$ & & 0.347 & & \\
\hline Preoperative albumin(g/L) & & 0.448 & & \\
\hline Neoadjuvant chemotherapy & Absent vs present & 0.233 & & \\
\hline Neoadjuvant radiotherapy & Absent vs present & 0.097 & $2.876(1.030-8.032)$ & 0.044 \\
\hline Surgical approach & Laparoscopy vs open & 0.129 & & \\
\hline Surgical method & Dixon vs Parks or Nose & 0.319 & & \\
\hline Anastomosis method & Stapled vs hand-sewn & 0.479 & & \\
\hline Operation time & & 0.016 & $4.314(0.972-19.157)$ & 0.055 \\
\hline Pathological T stage & $\mathrm{TI}$ and $\mathrm{T} 2$ vs $\mathrm{T} 3$ and $\mathrm{T} 4$ & 0.048 & $1.063(0.722-1.567)$ & 0.755 \\
\hline Pathological $\mathrm{N}$ stage & No vs NI vs N2 & 0.009 & $1.420(0.876-2.300)$ & 0.155 \\
\hline Metastasis & Absent vs present & 0.074 & $1.552(0.460-5.23 \mathrm{I})$ & 0.479 \\
\hline Distance from anal verge $(\mathrm{cm})$ & $>5$ vs $\leq 5$ & 0.327 & & \\
\hline
\end{tabular}




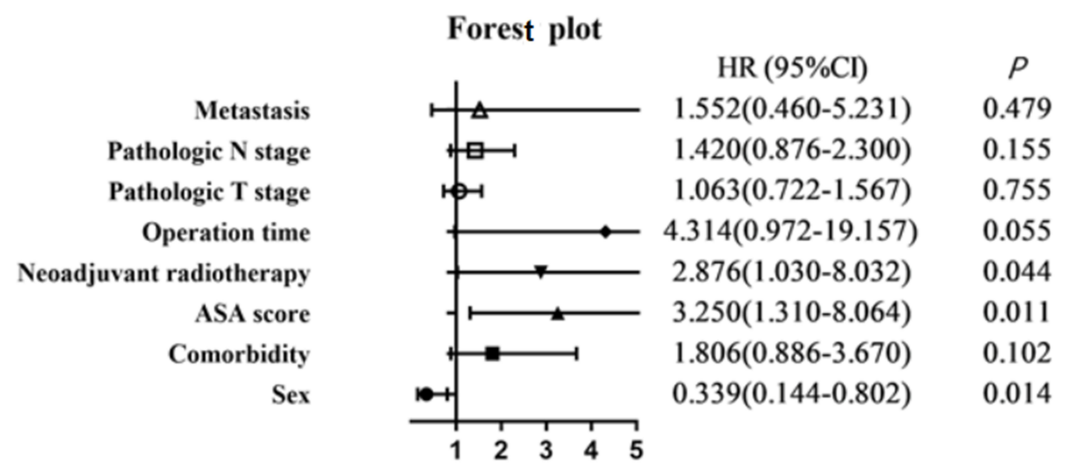

Figure I Forest plot.

showed that ASA score, male sex, and neoadjuvant radiotherapy influenced the incidence of AL (Figure 2). We plotted the sum of each variable on the total-point axis and obtained an estimated rate of AL by drawing a vertical line from the drawn total-point axis down to the result axis. ROC analysis showed that the nomogram had considerable predictive potential. The AUC of this model was 0.645 (Figure 3A). The C-index value of this model was 0.645 , indicating moderate predictive ability of the risk of AL. The calibration plot showed that the model was close to the ideal state, indicating good calibration (Figure 3B).

\section{Discussion}

We created a nomogram for predicting AL after rectal cancer surgery in elderly patients. Nomography for CRC hasthe advantage of accurate prediction of risk in patients. Our study retrospectively analyzed risk factors of AL for 326 consecutive elderly patients after rectal cancer and constructed a nomogram for predicting the incidence of AL. Previous reports have described many potential risk factors for AL after low anterior resection in rectal cancer. We found that ASA score $\geq 3$, male sex, and neoadjuvant radiotherapy were independent risk factors of $\mathrm{AL}$ on multivariate logistic regression analysis and developed a nomogram based on these three factors. The $\mathrm{C}$-index value of this model showed moderate predictive ability of the risk of AL. These findings may have clinical implications in the careful selection of elderly patients for ileostomy based on our nomogram. Previous reports have described many potential risk factors of AL after low anterior resection in rectal cancer. Hoshino et $\mathrm{al}^{17}$ reported a nomogram for predicting the risk of AL after anterior resection, which included sex, serum albumin, tumor location and diameter, and simultaneous resection of other organs. That study focused on low anterior resection. Yao et $\mathrm{al}^{21}$ collected tumor location, operation time, and preservation of the left colic artery, and created a nomogram for predicting the risk of AL after laparoscopic anterior resection. In contrast, our study focused on the rate of $\mathrm{AL}$ in elderly rectal cancer patients. To our knowledge, there have been few large-sample clinical studis on elderly rectal cancer patients and helping surgeons select precise surgical strategies.

The overall clinical AL rate was $19.33 \%$ in our study, comparable to previous research $(3 \%-21 \%){ }^{3}$ The significant difference in $\mathrm{AL}$ rates was due not only to the

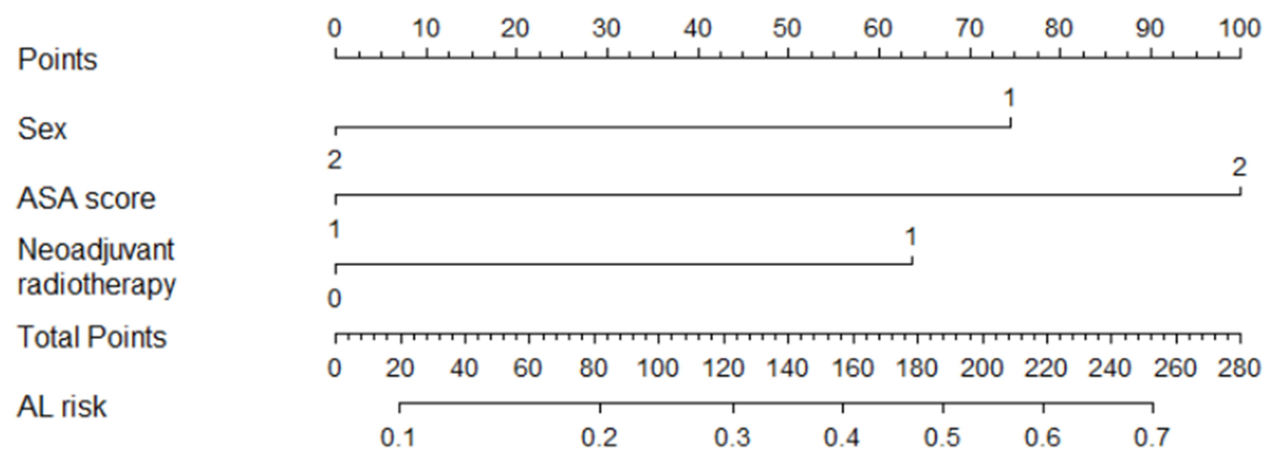

Figure 2 Nomogram for predicting anastomotic leakage ( $C$ index 0.645$)$ after rectal cancer surgery. Incidence of anastomotic leakage was estimated by summing scores of sex, ASA score, and neoadjuvant radiotherapy. 

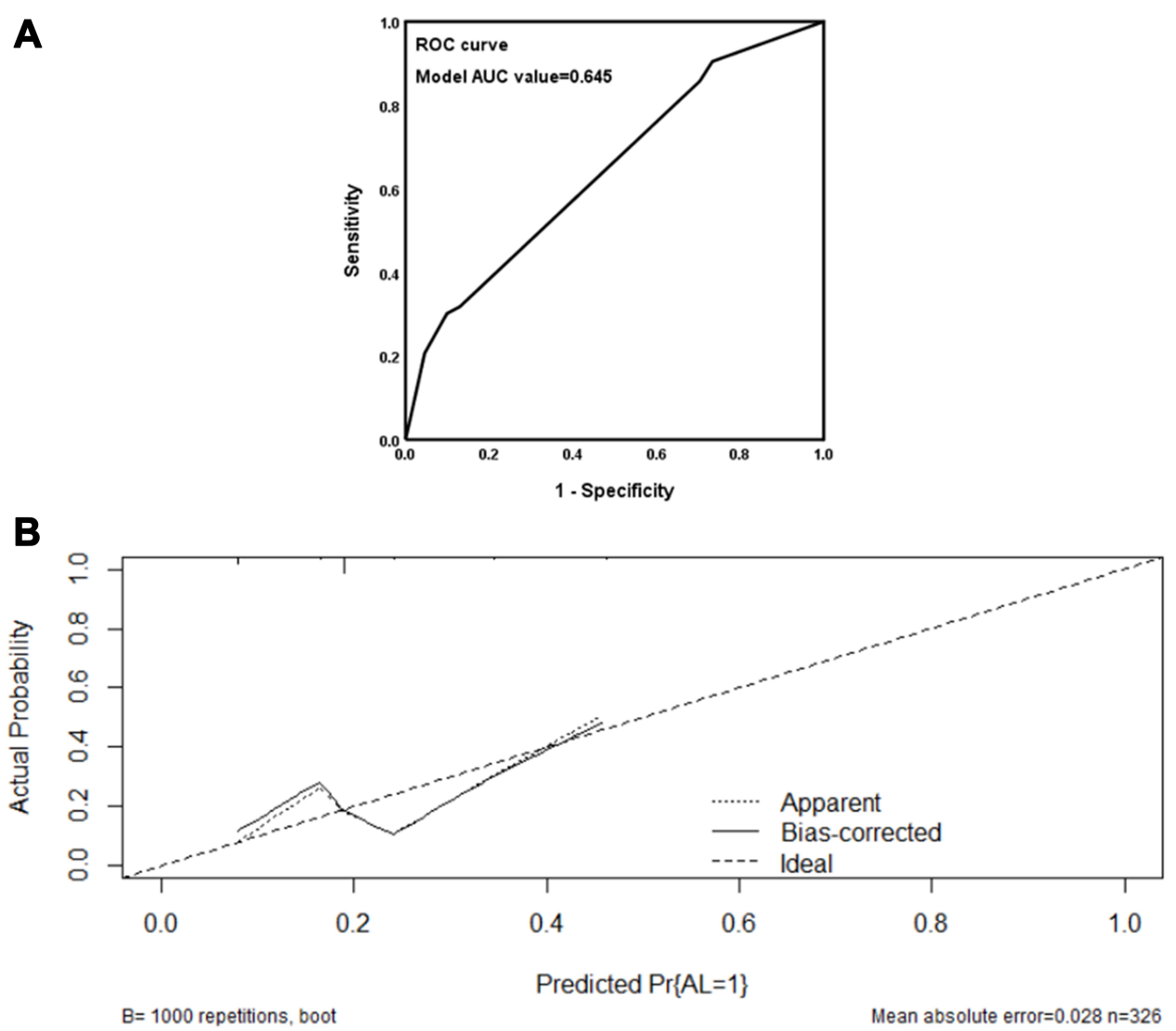

Figure 3 (A) ROC curve for the nomogram. AUC was 0.645 (95\% Cl $0.762-0.903)$. (B) Nomogram calibration curve. The $y$-axis represents the actual probability of anastomotic leakage. The $x$-axis represents predicted anastomotic leakage probability. The ideal line represents a perfect prediction model. The apparent line represents the performance of the nomogram, and a close fit to the ideal line represents a good prediction.

different ratios of participants who underwent rectal surgery but also the inconsistent inclusion criteria for AL. AL occurrence is affected by various factors, and the pathogenesis remains unclear. In previous studies, many factors have been found to be related to AL, such as age, sex, ASA score, BMI, preoperative chemoradiotherapy, tumor stage, tumor size, operative time, malnutrition, diabetes, and steroid treatment. ${ }^{15}$ However, for elderly patients with rectal cancer, there have been few studies to provide related risk factors for $\mathrm{AL}$ in large samples. We found that $\operatorname{sex}(P=0.014)$, ASA score $\geq 3 \quad(P=0.011)$, and neoadjuvant radiotherapy $(P=0.044)$ were independent risk factors of $\mathrm{AL}$. The risk of $\mathrm{AL}$ in male patients was 5.3-fold that of female patients in this study. The higher risk in men might be associated with a narrower pelvic space than female patients. ${ }^{22}$

As there was a significant difference between ASA scores of 1 or 2 and $\geq 3$, we classified ASA scores into these two categories. Our results were consistent with a previous study that reported an ASA score of at least 3 was independently related to a high risk of AL. ${ }^{15}$ Some studies have reported a significant association between neoadjuvant radiotherapy and $\mathrm{AL}$, similar to our results. $^{3,23}$ Neoadjuvant radiotherapy can not only kill tumor cells, but can also cause tissue inflammatory edema and fibrotic plaque formation at the radiotherapy site. Among the tumor features, lower tumor location has been reported to be an important risk factor of AL. In a previous study, anal verge distance $<7 \mathrm{~cm}$ was a risk factor associated with AL occurrence. Vignali et $\mathrm{al}^{24}$ showed that AL incidence was $8 \%$ when the tumor location was within $7 \mathrm{~cm}$ of the anal margin and $1 \%$ when it was $>7 \mathrm{~cm}$. There was no significant difference in tumor location between the two groups in our study, possibly due to different specific locations of upper and lower rectal cancer: we regarded $5 \mathrm{~cm}$ from the anal margin as the threshold. Future studies are required to investigate a more feasible number as a cutoff point. Tumor stage has also been reported as significant risk factor of AL after rectal cancer surgery. ${ }^{6}$ In our study, pathological $\mathrm{T}$ stage and pathological N stage were significantl related to AL occurrence, but not independent risk factors. 
We focused on elderly patients following rectal cancer resection, who have been reported to be at high risk of AL. We drew a nomogram based on the results of multivariate logistic regression analysis, including ASA score $\geq 3$, sex and neoadjuvant radiotherapy. Our nomogram can offer the surgeons an accurate approximation of AL risk after rectal cancer surgery in elderly patients before surgery, because it is composed of preoperative clinical findings. It is convenient and feasible to help surgeons make better surgical plans before surgery. When patients at higher risk of AL are identified by the nomogram before surgery, Hartmann's procedure or diverting stoma would be an ideal option for these patients. After surgery, delayed resumption of oral intake or removal of the drainage tube might be better. Also, these patients should be monitored carefully and more active interventions taken during the perioperative period.

The strength of our study was that it focused on the problem of $\mathrm{AL}$ in elderly patients following rectal cancer surgery, which has been little reported. We investigated multiple factors that may be associated with $\mathrm{AL}$ and constructed a nomogram to provide good prediction of AL risk before surgery. However, this study has several limitations. Firstly, it was a retrospective, single-center study with a relatively small sample, which has the limitation of selection bias with regard to different types of procedure. For example, the number of male patients ( $70.55 \%$ ) was much higher than female patients (29.35\%), which would lead to a higher overall clinical AL rate than the reported data. Secondly, it is still unclear whether the nomogram created in this study can be used by all surgeons, because its performance was not assessed in another cohort. We expect that a prospective large-sample and multicenter study will be conducted in future to improve the reliability and practicability of the prediction model.

\section{Conclusion}

This study screened out the risk factors of AL after rectal cancer surgery in elderly patients through multivariate analysis and constructed a nomogram to predict the possibility of AL. The nomogram showed good ability to predict $\mathrm{AL}$ and might be helpful in provide reference points for the selection of surgical procedures and perioperative treatment.

\section{Ethics Approval and Informed Consent}

The study was approved by the institutional ethics board of the Sixth Affiliated Hospital, Sun Yat-Sen University
(2020ZSLYEC-297). We confirm that we have obtained ethical approval to conduct the study and publish the data set. Data obtained contained no patient identifiers. Patient data was retrieved from our hospital database and there was no intervention, so patient consent was not required. This study complied with the Declaration of Helsinki.

\section{Acknowledgments}

This work was supported by the Science and Technology Program of Guangzhou (201803010074). Chuangkun Li, Weiwen Liang and Lili Chu are co-first authors. Rongkang Huang will handle correspondence, including any queries about methodology and materials.

\section{Disclosure}

The authors have no conflicts of interest to declare.

\section{References}

1. Siegel RL, Miller KD, Jemal A. Cancer statistics, 2018. CA Cancer J Clin. 2018;68:7-30. doi:10.3322/caac.21442

2. Benson AB, Venook AP, Al-Hawary MM, et al. NCCN guidelines insights: rectal cancer, version 6.2020. J Natl Compr Canc Netw. 2020;18:806-815. doi:10.6004/jnccn.2020.0032

3. Hu MH, Huang RK, Zhao RS, Yang KL, Wang H. Does neoadjuvant therapy increase the incidence of anastomotic leakage after anterior resection for mid and low rectal cancer? A systematic review and meta-analysis. Colorectal Dis. 2017;19:16-26. doi:10.1111/ codi. 13424

4. Kayano H, Nomura E, Ueda Y, et al. Short-term outcomes of OTSC for anastomotic leakage after laparoscopic colorectal surgery. Minim Invasive Ther Allied Technol. 2020;1-8. doi:10.1080/ 13645706.2020.1742743

5. Artus A, Tabchouri N, Iskander O, et al. Long term outcome of anastomotic leakage in patients undergoing low anterior resection for rectal cancer. BMC Cancer. 2020;20:780. doi:10.1186/s12885020-07109-4

6. Sciuto A, Merola G, De Palma GD, et al. Predictive factors for anastomotic leakage after laparoscopic colorectal surgery. World $J \quad$ Gastroenterol. 2018;24:2247-2260. doi:10.3748/wjg.v24. i2 1.2247

7. Kim CH, Lee SY, Kim HR, Kim YJ. Nomogram prediction of anastomotic leakage and determination of an effective surgical strategy for reducing anastomotic leakage after laparoscopic rectal cancer surgery. Gastroenterol Res Pract. 2017;2017:4510561. doi:10.1155/ 2017/4510561

8. Bostrom P, Haapamaki MM, Rutegard J, Matthiessen P, Rutegard M. Population-based cohort study of the impact on postoperative mortality of anastomotic leakage after anterior resection for rectal cancer. BJS Open. 2019;3:106-111. doi:10.1002/bjs5.50106

9. Jutesten H, Draus J, Frey J, et al. High risk of permanent stoma after anastomotic leakage in anterior resection for rectal cancer. Colorectal Dis. 2019;21:174-182. doi:10.1111/codi.14469

10. Hain E, Maggiori L, Manceau G, Zappa M, Prost ALDJ, Panis Y. Persistent asymptomatic anastomotic leakage after laparoscopic sphincter-saving surgery for rectal cancer: can diverting stoma be reversed safely at 6 months? Dis Colon Rectum. 2016;59:369-376. doi:10.1097/DCR.0000000000000568 
11. Montroni I, Ugolini G, Saur NM, et al. Personalized management of elderly patients with rectal cancer: expert recommendations of the European Society of Surgical Oncology, European Society of Coloproctology, International Society of Geriatric Oncology, and American College of Surgeons Commission on Cancer. Eur J Surg Oncol. 2018;44:1685-1702. doi:10.1016/j.ejso.2018.08.003

12. The Chinese Society of Colorectal Cancer. Expert consensus on perioperative management of elderly patients with colorectal cancer in China(2020 Edition). Chin J Colorect Dis. 2020;9:325-334.

13. Sparreboom CL, van Groningen JT, Lingsma HF, et al. Different risk factors for early and late colorectal anastomotic leakage in a nationwide audit. Dis Colon Rectum. 2018;61:1258-1266. doi:10.1097/DCR.0000000000001202

14. Zhou S, Zhou H, Zheng Z, Liang J, Zhou Z, Wang X. Predictive risk factors for anastomotic leakage after anterior resection of rectal cancer in elderly patients over 80 years old: an analysis of 288 consecutive patients. World J Surg Oncol. 2019;17:112. doi:10.1186/s12957-019-1655-Z

15. Parthasarathy M, Greensmith M, Bowers D, Groot-Wassink T. Risk factors for anastomotic leakage after colorectal resection: a retrospective analysis of 17,518 patients. Colorectal Dis. 2017;19:288-298. doi:10.1111/codi.13476

16. Eveno C, Latrasse V, Gayat E, Lo DR, Dohan A, Pocard M. Colorectal anastomotic leakage can be predicted by abdominal aortic calcification on preoperative CT scans: a pilot study. J Visc Surg. 2016;153:253-257. doi:10.1016/j.jviscsurg.2016.03.007

17. Hoshino N, Hida K, Sakai Y, et al. Nomogram for predicting anastomotic leakage after low anterior resection for rectal cancer. Int J Colorectal Dis. 2018;33:411-418. doi:10.1007/s00384-0182970-5
18. Waterland $\mathrm{P}, \mathrm{Ng} \mathrm{J}$, Jones A, et al. Using CRP to predict anastomotic leakage after open and laparoscopic colorectal surgery: is there a difference? Int J Colorectal Dis. 2016;31:861-868. doi:10.1007/ s00384-016-2547-0

19. Zhang G, Li R, Zhao X, Meng S, Ye J, Zhao L. Validation of the American Joint Committee on Cancer eighth edition staging system in patients undergoing hepatectomy for hepatocellular carcinoma: a US population-based study. J Surg Res. 2018;222:55-68. doi:10.1016/j.jss.2017.09.044

20. Rahbari NN, Weitz J, Hohenberger W, et al. Definition and grading of anastomotic leakage following anterior resection of the rectum: a proposal by the International Study Group of Rectal Cancer. Surgery. 2010;147:339-351. doi:10.1016/j.surg.2009.10.012

21. Yao HH, Shao F, Huang Q, Wu Y, Qiang ZZ, Liang W. Nomogram to predict anastomotic leakage after laparoscopic anterior resection with intracorporeal rectal transection and double-stapling technique anastomosis for rectal cancer. Hepatogastroenterology. 2014;61:1257-1261.

22. Park JS, Huh JW, Park YA, et al. Risk factors of anastomotic leakage and long-term survival after colorectal surgery. Medicine (Baltimore). 2016;95:e2890. doi:10.1097/MD.0000000000002890

23. Qin Q, Ma T, Deng Y, et al. Impact of preoperative radiotherapy on anastomotic leakage and stenosis after rectal cancer resection: post hoc analysis of a randomized controlled trial. Dis Colon Rectum. 2016;59:934-942. doi:10.1097/DCR.0000000000000665

24. Pakkastie TE, Luukkonen PE, Jarvinen HJ. Anastomotic leakage after anterior resection of the rectum. Eur J Surg. 1994;160:293-300.

\section{Publish your work in this journal}

Cancer Management and Research is an international, peer-reviewed open access journal focusing on cancer research and the optimal use of preventative and integrated treatment interventions to achieve improved outcomes, enhanced survival and quality of life for the cancer patient.
The manuscript management system is completely online and includes a very quick and fair peer-review system, which is all easy to use. Visit http://www.dovepress.com/testimonials.php to read real quotes from published authors. 